\title{
Blue sun reflected from water: optical lessons from observations of nature
}

Joseph Shaw, Michael Vollmer

Joseph A. Shaw, Michael Vollmer, "Blue sun reflected from water: optical lessons from observations of nature," Proc. SPIE 10452, 14th Conference on Education and Training in Optics and Photonics: ETOP 2017, 104523B (16 August 2017); doi: 10.1117/12.2270481

SPIE Event: 14th Conference on Education and Training in Optics and Photonics, ETOP 2017, 2017, Hangzhou, China 


\title{
Blue sun reflected from water: optical lessons from observations of nature
}

Joseph A. Shaw* ${ }^{\mathrm{a}}$, Michael Vollmer ${ }^{\mathrm{b}}$

${ }^{a}$ Electrical and Computer Engr. Dept., Montana State University, Bozeman, Montana, USA 59717

${ }^{\mathrm{b}}$ University of Applied Sciences Brandenburg, Magdeburgerstr. 50, 14470 Brandenburg, Germany

\begin{abstract}
Specular reflections of the sun from a wind-rippled water surface combine to form a glitter pattern. These reflections are expected to not alter the perceived color of the sun (or other light source), but when the light is reflected near the Brewster angle, the highly polarized glints can appear blue when observed through a polarizer or polarizing sun glasses. This blue appearance is a result of blue light leakage through a standard film polarizer oriented orthogonal to the plane of polarization of the reflected light. Measurements are shown of crossed-polarizer transmission spectra exhibiting blue and near infrared light leakage for photographic polarizing filters and polarized sunglasses. A variety of photographs are shown to confirm blue light leakage as the source of the blue glint color.
\end{abstract}

Keywords: Polarization, water optics, glints, optics education

\section{INTRODUCTION}

Much can be learned about optics by critically observing patterns of light and color in nature. ${ }^{1}$ A fairly common natural optical phenomenon is sun glitter, ${ }^{2}$ which is a collection of specular reflections of the sun (or "glints") on the surface of water, as shown in Fig. 1. Glitter patterns change shape depending on the water surface roughness and the solar elevation angle. ${ }^{3,4}$ Because the water surface is roughened by small, wind-driven waves, ${ }^{5,6,7}$ glitter pattern measurements can be used for remote sensing of wind patterns. ${ }^{7,9}$ Under very light-wind conditions the number of sun glints decreases and interesting fun-house-mirror kind of reflections of the surroundings can be seen, as shown in Fig. $2 .^{10}$ This is further illustrated in Fig. 3, which shows multiple sun reflections on the rough water surface and a single sun reflection on the smooth water within the enclosed pool in the foreground.

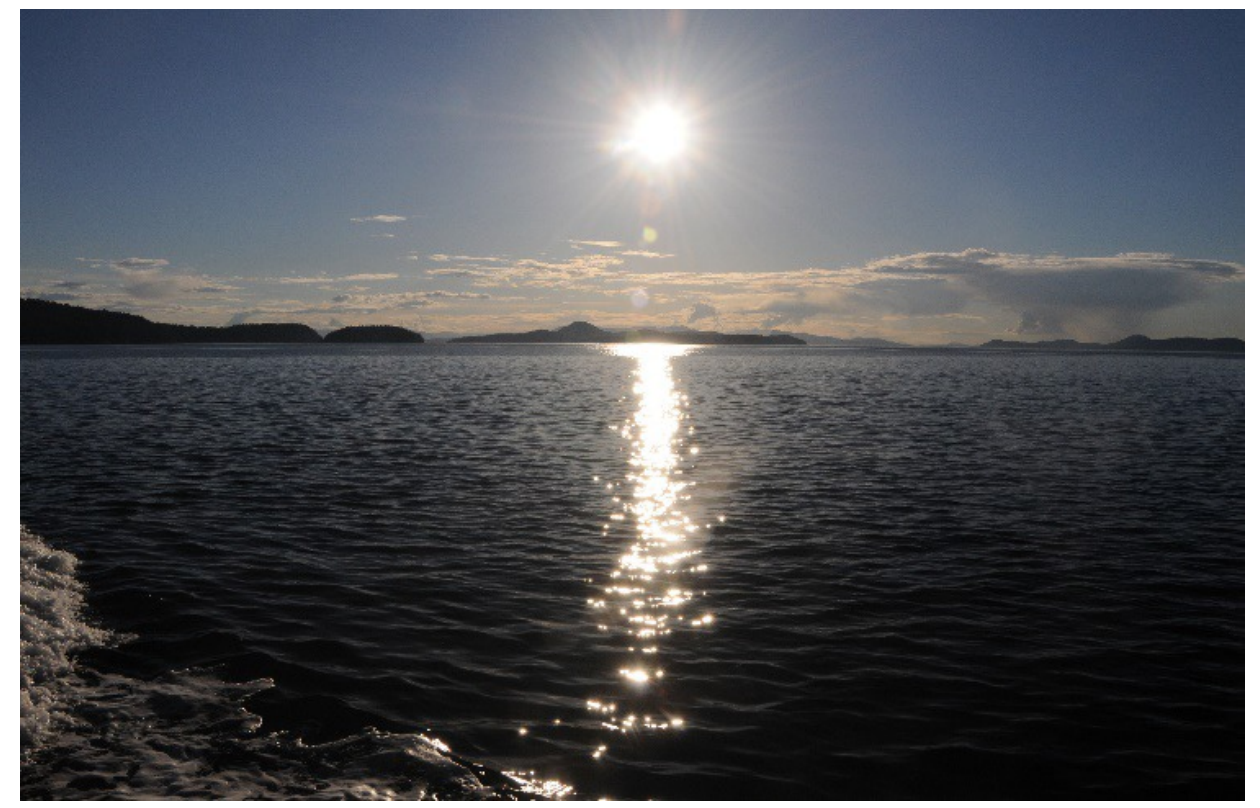

Figure 1. A sun glitter pattern contains numerous specular reflections of the sun from tilted wave surfaces.

*joseph.shaw@montana.edu; phone 1406 994-7261; fax 1406 994-2505; www.montana.edu/jshaw

14th Conference on Education and Training in Optics and Photonics: ETOP 2017, edited by Xu Liu, Xi-Cheng Zhang, Proc. of SPIE Vol. 10452, 104523B · (c) 2017 ICO, IEEE, OSA, SPIE

CCC code: $0277-786 X / 17 / \$ 18 \cdot$ doi: $10.1117 / 12.2270481$ 

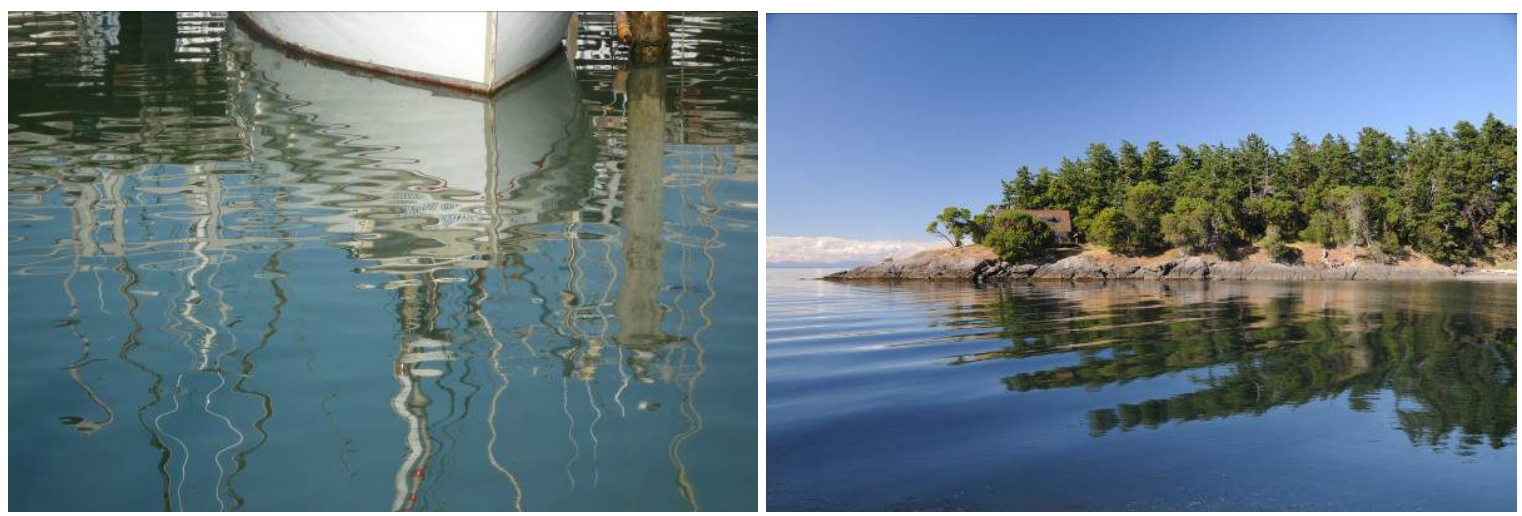

Figure 2. Examples of distorted reflections of the surroundings that can be seen on lightly undulating water surfaces.

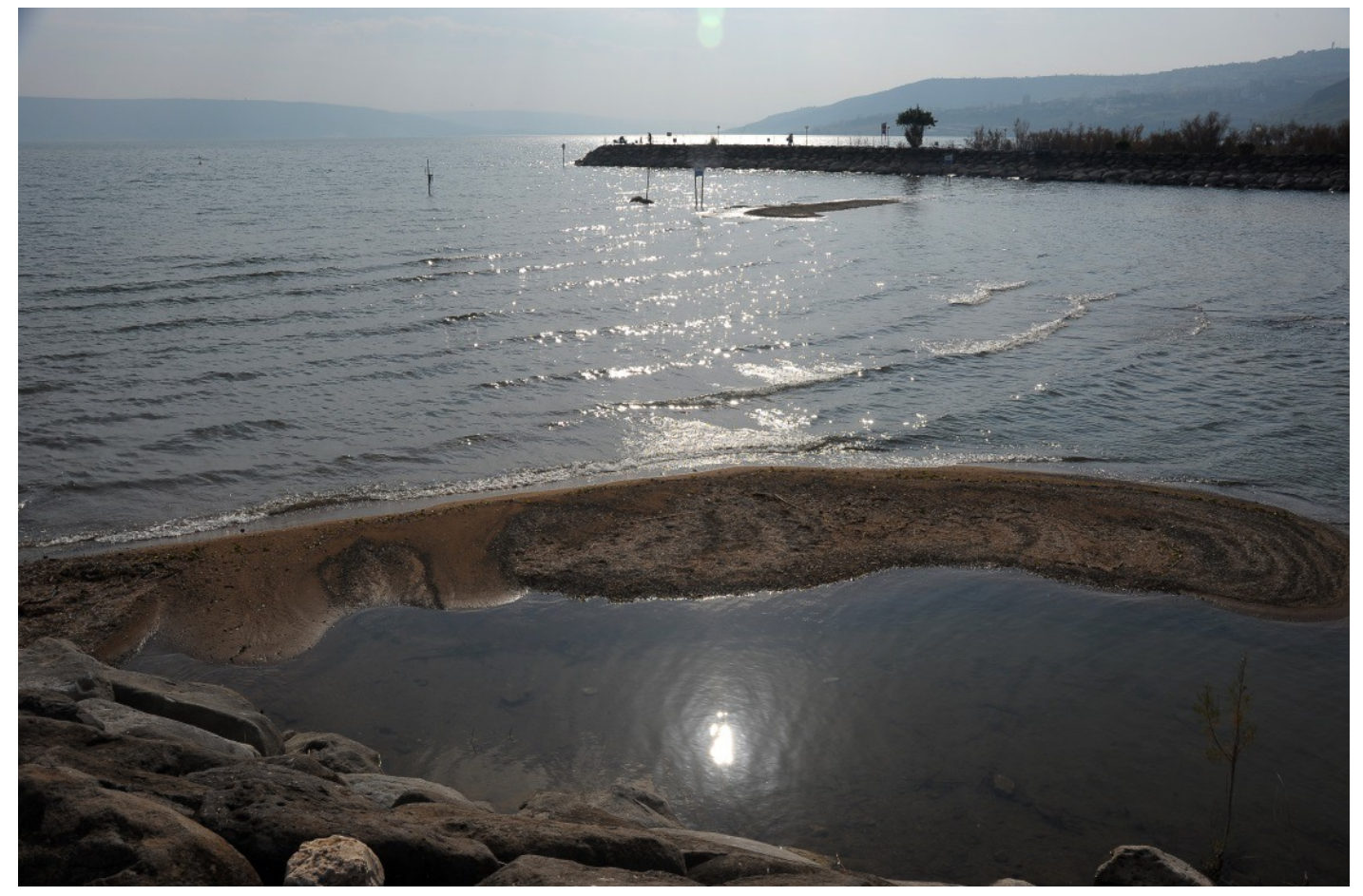

Figure 3. Multiple sun glints on wavy water surface and single sun reflection on smooth water.

The photographs in Fig. 4 show a glitter pattern close up so that the individual glints are easily discerned. Because we expected to see white light, i.e. colorless glints (or perhaps orange glints with a reddened setting sun ${ }^{11}$ ), we were surprised on several occasions to see blue glints when observing glitter patterns through polarizing sun glasses or through a polarizing filter oriented for minimum glint brightness, as shown in the right-hand photograph in Fig. 4 (the image exposures were 1/800 s at f/14 and ISO400 for the left-hand image and 1/80 s at f/5.6 and ISO400 for the righthand image). The same effect occurred for viewing through either a polarizing filter on a camera lens or polarized sunglasses in front of the lens. Similarly, the same effect occurred for both linear and circular polarizing filters. This is because circular polarizing filters are made of a linear polarizer on the object side and a quarter-wave plate on the camera side, so they act as a regular linear polarizer for detecting light from the scene. After some investigation, we found that the blue glint color arises because of a small amount of blue light leakage through the polarizing filter. ${ }^{2}$ In other words, the polarizer efficiency falls off at short wavelengths (it also falls off even more at near-infrared wavelengths, but that is not a significant factor here). Figures 5 and 6 show additional examples of blue glints - this time with a single sun reflection on a very smooth water surface viewed through a horizontal polarizer for the left-hand images and a vertical polarizer for the right-hand images. Fig. 5 is a wide-angle view, while Fig. 6 is a zoomed-in image centered on the sun reflection. 

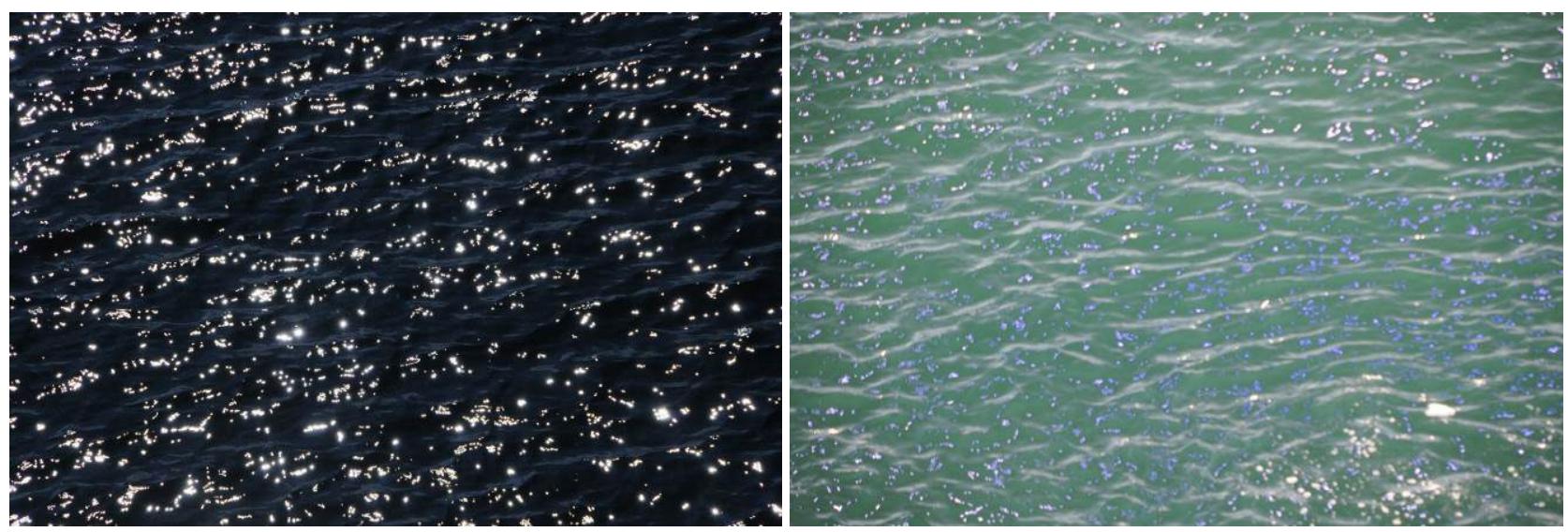

Figure 4. Sun glints observed through a polarizing filter: (left) polarizer oriented approximately horizontal for maximum glint brightness; (right) polarizer oriented approximately vertical for minimum glint brightness.
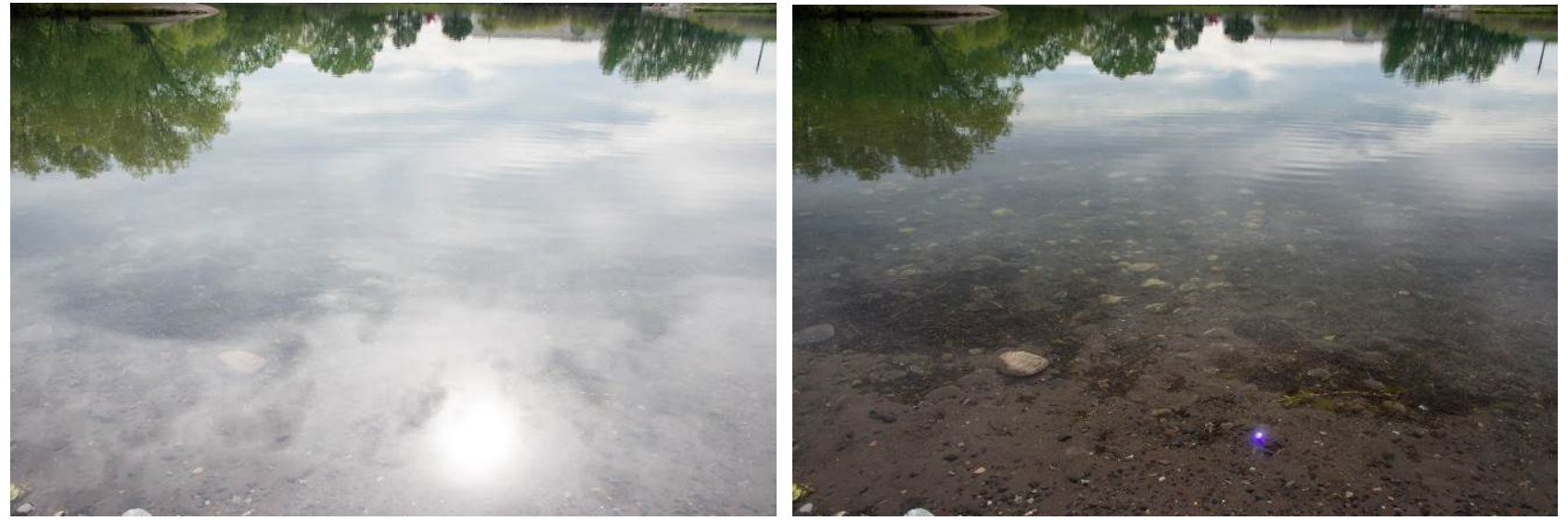

Fig. 5. Reflection from a calm lake surface with the sun $1.2^{\circ}$ from the Brewster angle at 1529 UTC, 19 May 2016, solar elevation angle $=35.8^{\circ}$ : (left) polarizer rotated for maximum glint brightness; (right) polarizer rotated for minimum glint brightness.
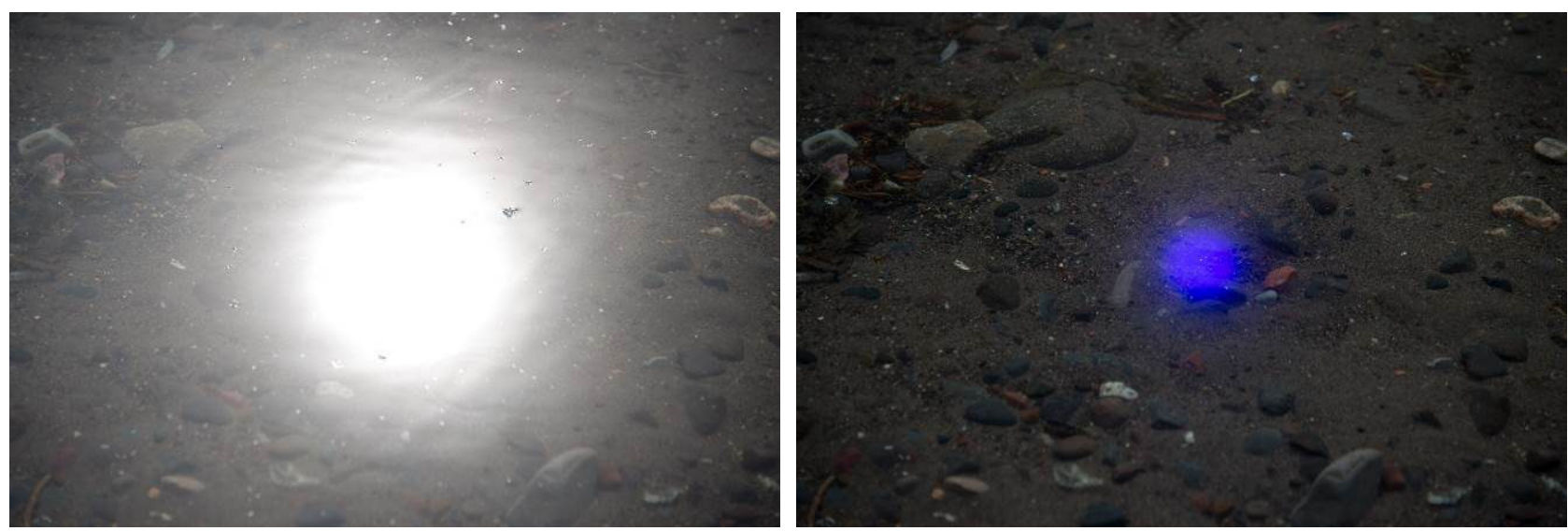

Fig. 6. Zoomed-in images of reflection on a calm lake surface with the sun $1.2^{\circ}$ from the Brewster angle at 1529 UTC, 19 May 2016 , solar elevation angle $=35.8^{\circ}$ : (left) polarizer rotated for maximum glint brightness; (right) polarizer rotated for minimum glint brightness. 


\section{GLINT POLARIZATION AND BLUE GLINTS}

Glints are partially polarized by reflection and become $100 \%$ polarized when the reflection angle is very near the Brewster angle (approximately $53^{\circ}$ ). ${ }^{12-14}$ This is indicated in the plots of Fig. 7 that show the s- and p-polarized reflectivity on the left and the corresponding degree of polarization ${ }^{15}$ on the right for red light reflected from water. Even if the surface is rippled by the wind, the glints remain at least $90 \%$ polarized for angles of $45^{\circ}-61^{\circ}$ and $80 \%$ polarized for angles of $41^{\circ}-65^{\circ}$; this allows for highly polarized glints even with modestly wind-rippled surfaces. ${ }^{10}$

The glints in the right-hand images of Figs. 4, 5, and 6 took on a blue appearance because they were observed through a polarizer whose transmission axis was oriented approximately vertical, which would be orthogonal to the axis of polarization for the reflected light in the glints. This occurred because the polarizer efficiency drops off for shortwavelength (blue) light, as shown in the transmission spectrum shown in the left-hand image of Fig. 8. This spectrum was recorded with an initially randomly polarized white light source shining through the polarizer under test followed by a second, high-quality, broadband polarizer with a contrast ratio near $10^{5}$ for $350-580 \mathrm{~nm}$ and at least $10^{3}$ for $580-2600$ $\mathrm{nm}$ (Meadowlark Optics OWL polarizer). This crossed-polarizer spectrum exhibits a very large light leak in the near infrared (NIR) beyond about $780 \mathrm{~nm}$, but this radiation is not visible by eye and is largely not recorded in standard visible-light cameras, which are fitted with NIR-blocking filters. ${ }^{16.17}$ Since the same blue glints were visible simply by looking through a polarizer or polarizing sunglasses with the naked eye, we quickly knew that the NIR leak was not the source of the blue color. The actual source of the blue color was the less-obvious blue light leak at wavelengths below $450 \mathrm{~nm}$. The blue light leak is well known in the community of people who design polarizers and electronic displays that incorporate polarizers, ${ }^{18}$ but many people are not aware of its existence.

An even larger version of the blue light leak is apparent in the crossed-polarizer spectrum shown in the right-hand frame of Fig. 8. This spectrum was measured for a pair of polarized sunglasses (Outlaw by Balzer). Blue glints should be visible with any type of polarized sunglasses, but this model makes it particularly easy to see blue glints. In fact, it is so simple that when wearing these glasses, we now regularly see blue colors in reflections from smooth floors, car windows, and just about any relatively smooth surface. Several examples are shown in Figures $8-13$, which are photographs taken with smartphone cameras looking through the Balzer sunglasses.
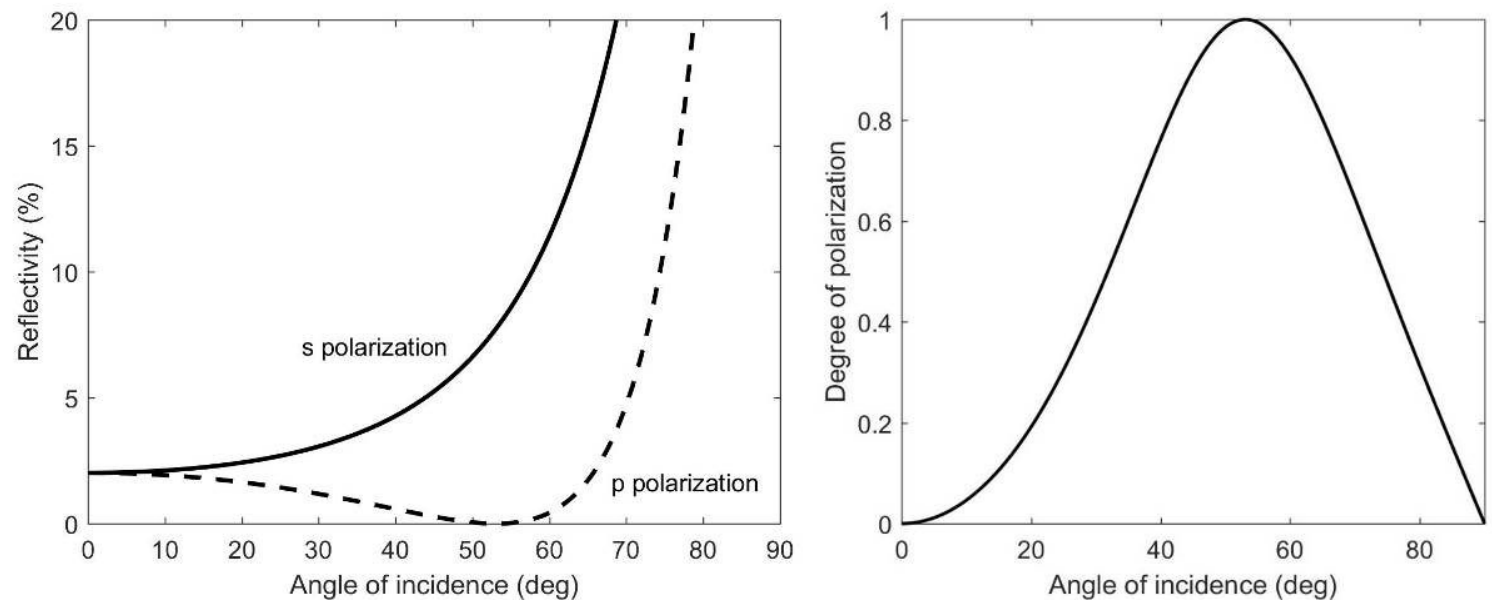

Figure 7. Light becomes partially polarized on reflection from water: (left) reflectivity of water for red light; (right) degree of polarization, reaching a value of 1 that indicates $100 \%$ polarized light at the Brewster angle of approximately $53^{\circ}$. 

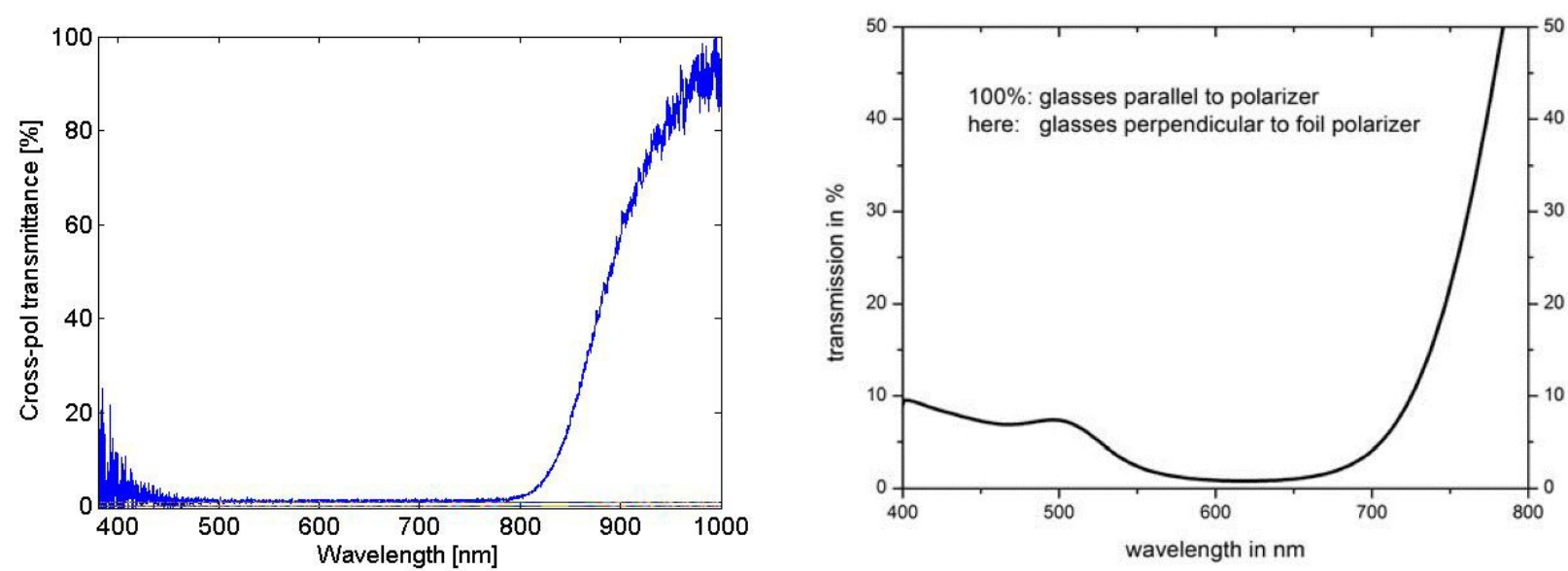

Fig. 8. Transmission spectra of (left) a standard film polarizer and (right) polarized sunglasses - both oriented with their transmission axis oriented orthogonal to the axis of a second broadband polarizer.

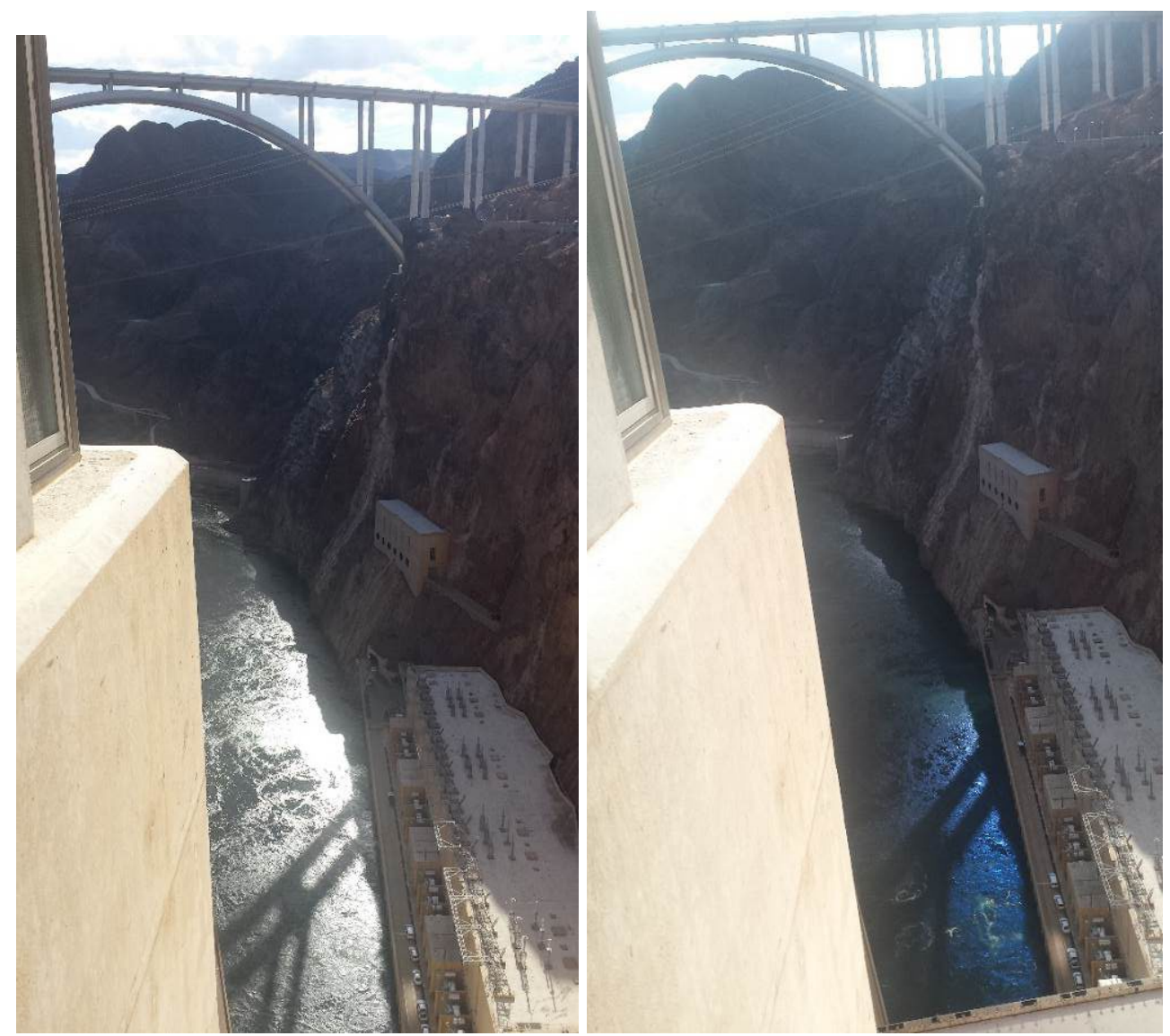

Fig. 9. Smartphone photographs of sun glint on the Colorado River behind the Hoover Dam: (left) no polarizer; (right) looking through Balzer sunglasses oriented to minimize glint brightness. 

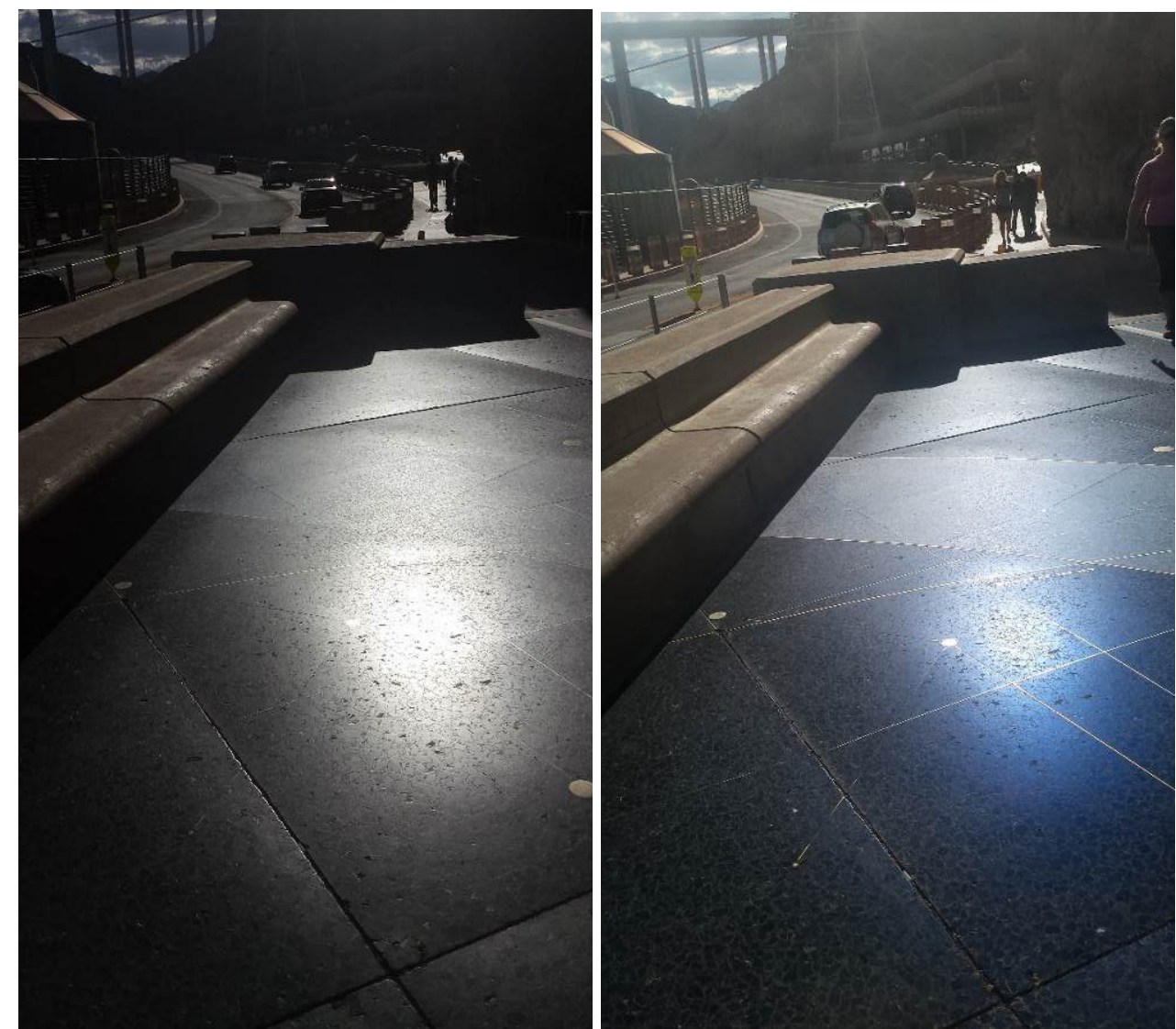

Fig. 10. Smartphone photographs of sun glint on a marble floor near the Hoover Dam monument: (left) no polarizer; (right) looking through Balzer sunglasses oriented to minimize glint brightness.

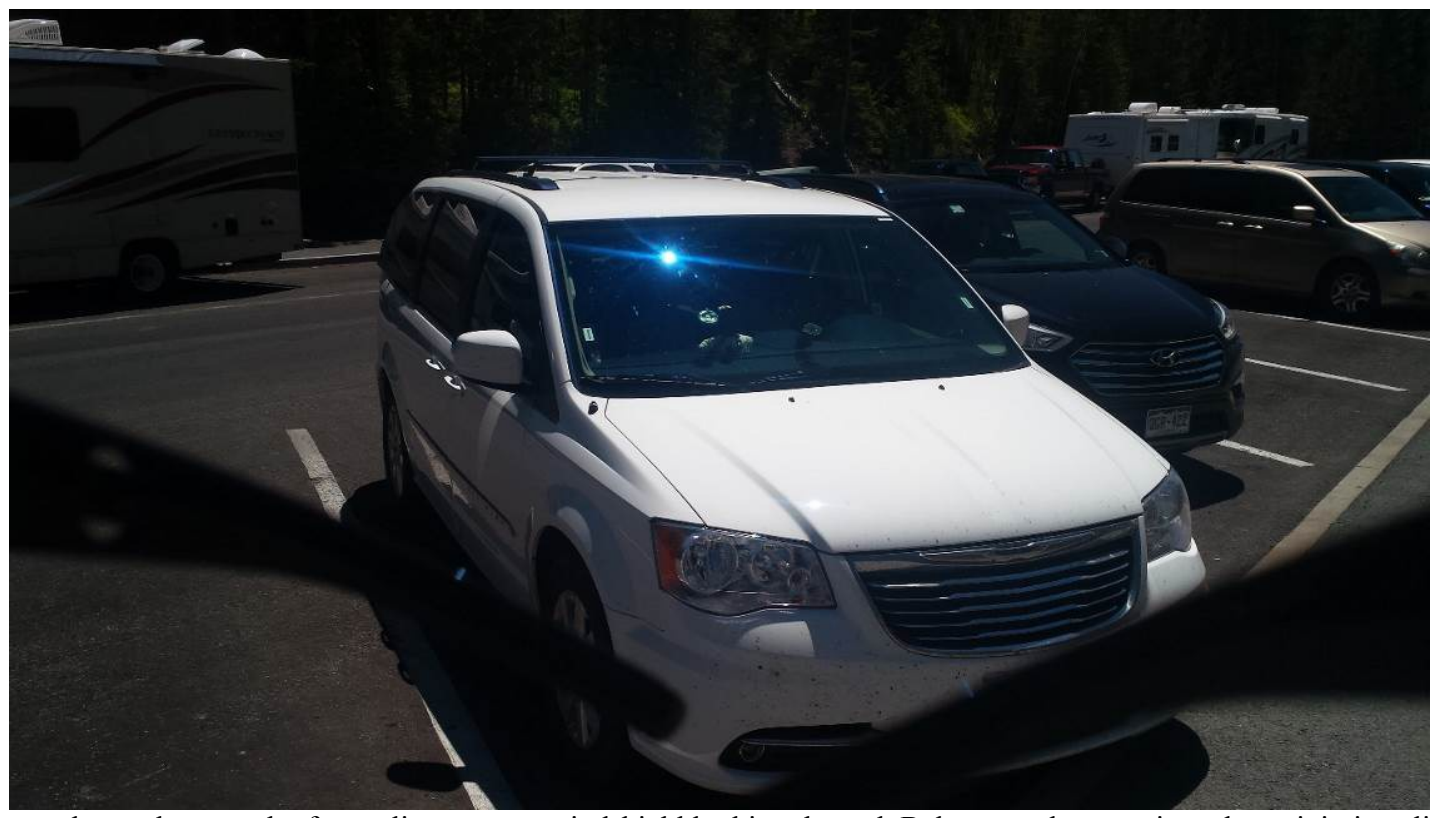

Fig. 11. Smartphone photograph of sun glint on a car windshield looking through Balzer sunglasses oriented to minimize glint brightness. 


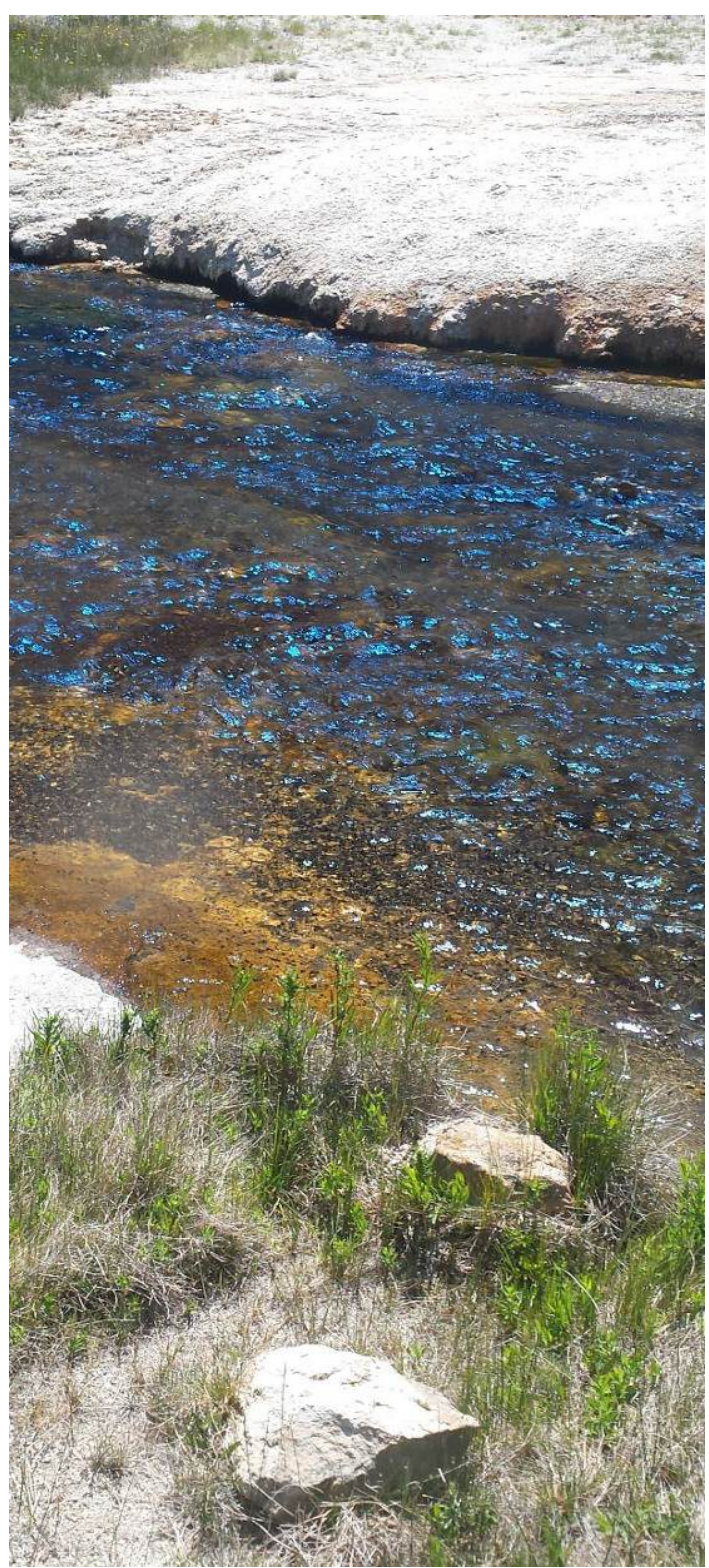

Fig. 12. Smartphone photograph of sun glint on Iron Spring Creek next to Black Sand Basin at Yellowstone National Park, looking through Balzer sunglasses oriented to minimize glint brightness.

Proc. of SPIE Vol. 10452 104523B-7 


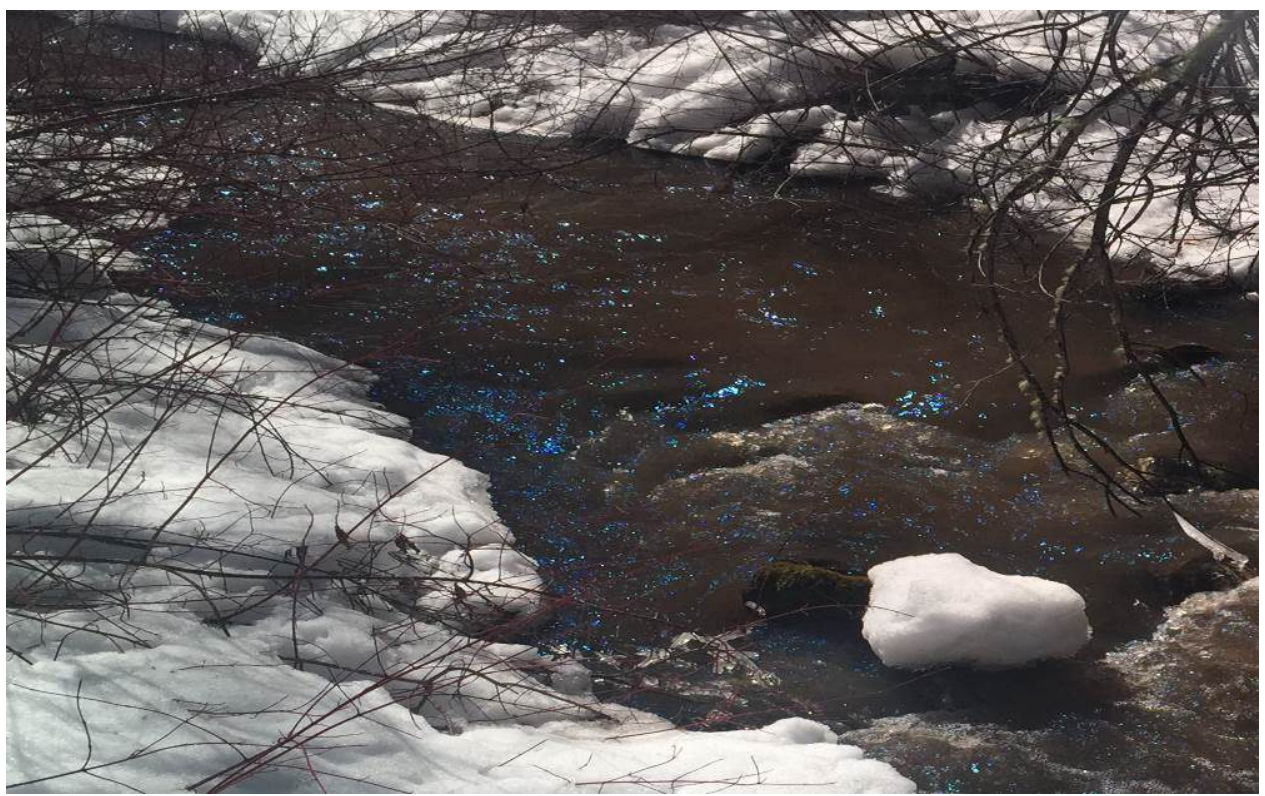

Fig. 13. Smartphone photograph of sun glint on Bear Creek near Bozeman, MT, looking through Balzer sunglasses oriented to minimize glint brightness.

\section{CONCLUSION}

Interesting lessons about optics can be learned and taught using simple polarizing filters or sunglasses and cameras. We have shown crossed-polarizer transmission spectra measured for polarized sunglasses and for photographic polarizing filters, both of which exhibited blue light leakage (as well as NIR leakage). This blue light leakage results in blue reflections when the reflected light occurs at or near the Brewster angle and is viewed through a polarizer oriented with its axis orthogonal to the reflected light polarization. Such blue reflections can be seen on water, car windshields, smooth marble floors, and other relatively smooth surfaces. Due to the relative ease of observation and the low cost of polarizing sunglasses, the optics of blue sun glints and glitter path can easily be added to physics courses and included as homework for optics students.

\section{REFERENCES}

[1] Shaw, J. A., [Optics in the Air: Observing optical phenomena through airplane windows], SPIE Press, Bellingham, WA (2017).

[2] Shaw, J. A. and M. Vollmer, "Blue glints on water viewed through a polarizer," Appl. Opt. 56(19), G36-G41 (2017).

[3] Shaw, J. A., "Glittering light on water," Optics and Photonics News 10(3), 43-45, 68 (1999).

[4] Lynch, D. K., Dearborn, D. S., and Foley, J. A., "Glitter and glints on water," Appl. Opt. 38(28), F39-49 (2011).

[5] Shaw, J. A. and Churnside, J. H., "Scanning-laser glint measurements of sea-surface slope statistics," Appl. Opt. 36(18) 4202-4213 (1997).

[6] Shaw, J. A. and Churnside, J. H., "Fractal laser glints from the ocean surface," J. Opt. Soc. Am. A 14(5), 1144-1150 (1997).

[7] Cox, C. and Munk, W., "Measurement of the roughness of the sea surface from photographs of the sun's glitter," J. Opt. Soc. Am. 44(11), 838-850 (1954).

[8] Levanon, N., "Determination of the sea surface slope distribution and wind velocity using sun glitter viewed from a synchronous satellite," J. Phys. Oceanography 1, 214-220 (1971).

[9] Wald, L. and Monget, J.-M., "Sea surface winds from sun glitter observations," J. Geophys. Res. 88(C4), 2547-2555 (1983).

[10] Walsh, E. J., Banner, M. L., Churnside, J. H., Shaw, J. A., Vandemark, D. C., Wright, C. W., Jensen, J. B., and Lee, S., "Visual demonstration of three-scale sea-surface roughness under light wind conditions," IEEE Trans. Geosci. Rem. Sens. 43(8), 1751-1762 (2005). 
[11] Vollmer, M. and Gedzelman, S. D., "Colours of the Sun and Moon: the role of the optical air mass," Eur. J. Phys. 27(2), 27(2), 299-310 (2006).

[12] Shaw, J. A., "Degree of linear polarization in spectral radiances for water-viewing infrared radiometers," Appl. Opt. 38(15), 3157-3165 (1999).

[13] Shaw, J. A., Descour, M. R., Sabatke, D. S., Garcia, J. P., Dereniak, E. L., "Measurements of midwave and longwave infrared polarization from water," Proc. SPIE 3754, 118-125 (1999.

[14] Shaw, J. A., "A survey of infrared polarization in the outdoors," Proc. SPIE 6660, 666006 (2007).

[15] Tyo, J. S., Goldstein, D. L., Chenault, D. B., and Shaw, J. A., "Review of passive imaging polarimetry for remote sensing applications," Appl. Opt. 45(22), 5453-5469 (2006).

[16] Mangold, K., Shaw, J. A., and Vollmer, M., “The physics of near-infrared photography,” Eur. J. Phys. 34(6), S51-S71 (2013).

[17] Vollmer, M. and Shaw, J. A., "Atmospheric optics in the near infrared," Appl. Opt. 56(19), G145-G155 (2017).

[18] Gunning, W. J. and Foschaar, J., "Improvement in the transmission of iodine-polyvinyl alcohol polarizers," Appl. Opt. 22(20), 3229-3231 (1983). 